\title{
Transdermal estradiol as a novel off-label treatment for Peyronie's disease: A case report
}

Jessie Thuswaldner ${ }^{1}$, J. Paul Fedoroff ${ }^{1,2}$

${ }^{1}$ University of Ottawa, Faculty of Medicine

${ }^{2}$ University of Ottawa, Department of Psychiatry

\section{ABSTRACT}

Introduction: This is a single-patient case report of a 75-year old married male with long-standing Peyronie's disease (PD). The patient's main symptoms were painful erections as well as secondary anxiety and depression. The symptoms could not be resolved through standard treatments without considerable side effects.

Objectives: To describe a patient with PD, whose persistently painful erections and psychologic distress were eliminated by a novel off-label treatment involving transdermal estradiol. This report also aims to present a novel off-label treatment for patients with paraphilias.

Methods: An interview with the patient, chart review, and a literature search were conducted. Informed consent was obtained from the patient and this report was approved by the Research Ethics Committee at the Royal Hospital in Ottawa.

Results: Treatment with transdermal estradiol decreased the patient's sex drive and virtually eliminated his erections with no undesirable side effects. The patient and his wife still enjoy sexual relations without the need for penile erection.

Conclusions: This case report presents a novel off-label treatment for PD. It also introduces a novel off-label method to treat men who wish to decrease their sex drive (e.g. men with paraphilic disorders). Replication of this treatment intervention in men with PD and new studies of its use in men with paraphilic disorders are warranted.

\section{RÉSUMÉ}

Introduction: This is a single-patient case report of a 75-year old married male with long-standing Peyronie's disease (PD). The patient's main symptoms were painful erections as well as secondary anxiety and depression. The symptoms could not be resolved through standard treatments without considerable side effects.

Objectives: To describe a patient with PD, whose persistently painful erections and psychologic distress were eliminated by a novel off-label treatment involving transdermal estradiol. This report also aims to present a novel off-label treatment for patients with paraphilias.

Methods: An interview with the patient, chart review, and a literature search were conducted. Informed consent was obtained from the patient and this report was approved by the Research Ethics Committee at the Royal Hospital in Ottawa.

Results: Treatment with transdermal estradiol decreased the patient's sex drive and virtually eliminated his erections with no undesirable side effects. The patient and his wife still enjoy sexual relations without the need for penile erection.

Conclusions: This case report presents a novel off-label treatment for PD. It also introduces a novel off-label method to treat men who wish to decrease their sex drive (e.g. men with paraphilic disorders). Replication of this treatment intervention in men with PD and new studies of its use in men with paraphilic disorders are warranted.

Keywords: Peyronie's disease, Treatment, Case Report, Men's Health

\section{INTRODUCTION}

Peyronie's disease (PD), a condition affecting up to $9 \%$ of men, is an abnormal curvature of the penis caused by excessive deposition of fibroid plaques in the tunica albuginea $(1,2)$. Common symptoms include chronic sexual dysfunction and mild-to-moderate pain during erections (3). The negative impact of PD on mood and quality of life is also well documented, with many PD patients suffering from mood/anxiety disorders, low self-esteem, emotional distress, etc., all of which may hinder an individual from seeking out relationships and lead to social isolation and stigmatization $(2,4,5)$.

The clinical course of PD typically consists of both an acute and a chronic phase. The acute phase, also known as the inflammatory phase, is characterized by erectile 
pain and progression of penile curvature (6). The chronic phase occurs after the first 6-18 months, during which the pain will disappear in more than $90 \%$ of patients and the curvature will stabilize $(1,3)$.

Treatment of PD can involve both surgical and/or nonsurgical interventions. Surgical options, which are most suitable once the progression of the curvature has stabilized, include plication, grafting, and penile prosthesis, with the latter being the gold standard for definitive treatment (2, 7). In terms of non-surgical interventions, intralesional drug injections such as collagenase clostridium histolyticum $(\mathrm{CCH})$, interferon alfa $2 \mathrm{~b}$, and verapamil may be used during the acute phase in order to slow the progression of the curvature (7-10).

Here, we report an unusual case of PD, in which the patient's persistently painful erections and psychological distress were relieved through treatment with transdermal estradiol.

\section{CASE REPORT}

In November 2009, a 64-year old Caucasian married gentleman presented to the Sexual Behaviour Clinic at the Royal Hospital in Ottawa with the desire to decrease his sex drive and frequency of penile erections. The patient, otherwise healthy, had been suffering with PD since May 2002 and had been prescribed several standard urologic treatments. A trial of biweekly intralesional verapamil injections over the course of eight months had minimal effects on curvature and pain. In April 2004, surgical plication had moderately improved his penile curvature but did not decrease the painful erections, which the patient described as a "sharp and intense pain". He had also developed secondary anxiety and depression. He was referred to the Sexual Behaviours Clinic when he declined a proposed penile implant.

The patient voluntarily consented to a treatment trial with oral medroxyprogesterone acetate (MPA), known by the brand name "Provera", which helped to decrease both his sex drive and frequency of erections. However, after taking MPA for three months, the patient reported the debilitating side effects of fatigue, gastrointestinal (GI) problems, paresthesia and weakness in arms and legs, dizziness (including tripping and falling), and episodes of "blurry vision". Changing dosage and dosage intervals did not help. The patient elected to discontinue treatment with
MPA in November 2016.

In August 2017, the patient was prescribed 1.25 grams of EstroGel, a $0.06 \%$ transdermal estradiol gel. The patient experienced a reduction in sex drive and erections just as he did with MPA, but without the intolerable side effects. The patient reported a greatly improved psychological state, with less stress and anxiety in regard to his sexuality, described as an inner "mental peace".

The dosage of transdermal estradiol gel was increased to 2.5 grams in August 2018, which further decreased the patient's sex drive and caused the desirable side effect of virtually eliminating his erections. Unlike MPA, the patient did not experience any undesirable side effects. Since then the patient and his wife have continued to be completely satisfied with the treatment which he wishes to continue.

\section{DISCUSSION}

While most patients with PD experience pain during the acute phase of the illness, this symptom is typically selflimited (1). As such, PD treatments tend to target penile curvature with the goal of restoring sexual functionality as opposed to alleviating the pain alone (3). Therefore, the treatment presented in this report may be useful for a patient during the painful (acute) stage of the disease, or for cases where the pain persists on a more chronic basis.

In addition, pain may preclude patients from benefiting from the standard treatments of PD. Since pain is generally indicative of active disease progression, it is considered a relative contraindication to surgery $(6,11) . \mathrm{CCH}$ injections, the only FDA approved non-surgical treatment for PD, work by degrading the collagen type I and II fibres in the fibroid plaques, and have been shown to reduce curvature. However, the presence of painful plaques is known to be characteristic of patients with a poor treatment response (7). Therefore, a treatment that targets pain reduction such as this one may allow patients to become eligible for other treatments in the future, but more research is required to explore this further.

It is important to acknowledge that use of transdermal estradiol as a monotherapy does not exclusively decrease the pain during erections, but decreases erections all together as well as sex drive, which would likely be undesirable and distressing for some men. However, these may be suitable outcomes for those suffering from severe 
The implications of this case are numerous, as they extend not only to other patients with PD, but also to any patients seeking to decrease sex drive and/or frequency of erections, such as patients convicted of sexual offense and men with problematic paraphilias. Current treatment for these patients involves psychotherapy with or without use of pharmacological therapy, such as hormone therapy, antiandrogens, gonadotropin-releasing hormone analogs, and selective serotonin reuptake inhibitors. Use of MPA for treatment of paraphilias is common, but its poor side effect profile often decreases compliance (12). Drugs that lower testosterone are the mainstay of pharmacotherapy for these patients, but it is important to consider the longterm dangers of lowering testosterone, such as increased risk of osteoporosis (13).

Use of oral estradiol to treat patients convicted of sexual offense has been previously investigated, but side effects such as nausea, vomiting, cardiac disturbances, and feminization (gynecomastia, redistribution of body fat, etc.) have limited its long-term success (14). Transdermal estradiol, which does not have the cardiovascular side effects of oral estradiol, presents a novel off-label treatment for both PD and paraphilias. There is no published study to our knowledge that uses transdermal estradiol in PD, although studies testing its application in men with prostate cancer have successfully lowered PSA levels and have not elicited significant side effects other than gynecomastia $(15,16)$.

In contrast to oral estradiol, transdermal estradiol avoids first-pass metabolism by the liver, which is thought to explain why its side effects are minimal compared to those of oral estradiol (17). There are many health benefits of transdermal estradiol that have made it a widely-used hormone therapy agent in postmenopausal women, such as relief from mood swings, vaginal dryness, and night sweats, as well as decreased risk of coronary artery disease and preservation of bone density (18). The latter two benefits have also been observed in male patients, but further research is warranted in order to better characterize use of transdermal estradiol in men (15).

\section{CONCLUSION}

Transdermal estradiol as demonstrated in this case, is a novel off-label treatment for PD, specifically in treating the symptoms of pain and secondary psychological distress.
Future research should investigate whether transdermal estradiol may be used for the off-label purpose of voluntarily curtailing erections in patients convicted of sexual offense and men with problematic paraphilias.

\section{REFERENCES}

1. Hussein AA, Alwaal A, and Lue TF. All about Peyronie's disease. Asian J Urol. 2015; 2(2):70-78.

2. Bella AJ, Lee JC, Grober ED, Carrier S, Benard F, Brock GB. Canadian Urological Association guideline for Peyronie's disease and congenital penile curvature. Can Urol Assoc J. 2018; 12(5):197-209.

3. Shaw EJ, Mitchell GC, Tan RB, Sangkum P, and Hellstrom WJ. The non-surgical treatment of Peyronie disease: 2013 update. World J Mens Health. 2013; 31(3):183-92.

4. Levine LA. The clinical and psychosocial Impact of Peyronie's Disease. Am J Manag Care. 2013; 19(4):55-61.

5. Terrier JE, Nelson CJ. Psychological aspects of Peyronie's disease. Transl Androl Urol. 2016; 5(3):290-295

6. Love C, Katz DJ, Chung E, Shoshany O. Peyronie's disease - Watch out for the bend. AFP. 2017; 46(9):655-659.

7. Capoccia E, Levine LA. Contemporary Review of Peyronie's Disease Treatment. Curr Urol Rep. 2018; 19(7):51.

8. Saju PR, Vikas V, Gopi P, Kauruv RS. Comparative study of oral and intralesional verapamil in Peyronie's Disease. Evid Based Med Healthc. 2017; 4(38):2300-2303.

9. Stewart CA, Yafi FA, Knoedler M, Mandava SH, McCaslin $I R$, Sangkum $P$, et al. Intralesional Injection of Interferon- $a 2 b$ Improves Penile Curvature in Men with Peyronie's Disease Independent of Plaque Location. J Urol. 2015; 194(6):17041707.

10. Wayne GF, Cordon BH. Contemporary surgical and nonsurgical management of Peyronie's disease. Transl Androl Urol. 2018; 7(4):603-617.

11. Levine LA, Larsen SM. Surgery for Peyronie's disease. Asian J Androl. 2013; 15(1):27-34.

12. Thibaut F, De La Barra F, Gordon H, Cosyns P, and Bradford JM. The World Federation of Societies of Biological Psychiatry (WFSBP) guidelines for the biological treatment of paraphilias. World J Biol Psychiatry. 2010; 11(4):604-655.

13. Mohamad NV, Soelaiman IN, Chin KY. A concise review of testosterone and bone health. Clin Interv Aging. 2016; 11:1317-1324

14. Bancroft J, Tennent G, Loucas K, et al. The control of deviant sexual behaviour by drugs: Behavioural changes following oestrogens and anti-androgens. $\mathrm{Br} \mathrm{J}$ Psychiatry. 1974; 125(0):310-315.

15. Gilbert DC, Duong T, Sydes M, Bara A, Clarke N, Abel P, et al. Transdermal oestradiol as a method of androgen suppression for prostate cancer within the STAMPEDE trial platform. BJU Int. 2018; 121(5):680-683.

16. Thibaut F, Cosyns P, Fedoroff JP, Broken P, Goethals K Bradford JMW. The world federation of societies of biological psychiatry (WFSBP) 2020 guidelines for the pharmacological treatment of paraphilic disorders. 2020 (In press). World $\mathrm{J}$ Biol Psychiatry.

17. Levin VA, Jiang $X$, Kagan $R$. Estrogen therapy for osteoporosis in the modern era. Osteoporosis Int. 2018; 29(5):1049-1055.

18. Beck KL, Anderson MC, Kirk JK. Transdermal estrogens in the changing landscape of hormone replacement therapy. Postgrad Med. 2017; 129:632-636. 